\title{
REVISTA RESIDÊNCIA PEDIÁTRICA E AS REDES SOCIAIS
}

\author{
Pediatric Residency journal and social networks
}

\author{
Bruna Brasil Seixas Bruno' \\ Sociedade Brasileira de Pediatria \\ Marilene Augusta Rocha Crispino Santos ${ }^{2}$ \\ Clemax Couto Sant'Anna ${ }^{3}$ \\ Universidade Federal do Rio de Janeiro \\ Marcia Garcia Alves Galvão4 \\ Leonardo Santos Martes ${ }^{5}$ \\ Sociedade Brasileira de Pediatria
}

\section{COMO CITAR}

BRUNO, Bruna Brasil Seixas. Revista Residência Pediátrica e as redes sociais. In: ABEC MEETING, 2019, Fortaleza. Anais... São Paulo: Associação Brasileira de Editores Científicos, 2019. p. 1-7. http://dx.doi.org/10.21452/abecmeeting. 2019. 208

\section{RESUMO}

A revista Residência Pediátrica (RP)* é uma publicação eletrônica quadrimestral da Sociedade Brasileira de Pediatria (SBP), online desde 2011, destinada aos médicos residentes da área de pediatria e afins, visando contribuir para a formação acadêmica dos residentes e incentivando-os a uma participação ativa na produção científica. Esse artigo objetivou apresentar um histórico sobre a RP, bem como investigar os periódicos nacionais e internacionais relacionados à pediatria e inseridos na plataforma CAPES quanto ao uso de redes sociais como o Facebook,

\footnotetext{
${ }^{1}$ Graduada em Biblioteconomia e Gestão de Unidades de Informação pela Universidade Federal do Rio de Janeiro (UFRJ). Pós-graduação em Comunicação e Marketing em Mídias Digitais pela Universidade Estácio de Sá.

2 Doutora em Medicina (Pesquisa Clínica) pela Universidade Federal do Rio de Janeiro (UFRJ).

${ }^{3}$ Professor do Departamento de Pediatria da Faculdade de Medicina da Universidade Federal do Rio de Janeiro (UFRJ)

${ }^{4}$ Doutora em Medicina (Saúde da Criança e do Adolescente) pela Universidade Federal do Rio de Janeiro (UFRJ

${ }^{5}$ Graduado em Comunicação Social/Jornalismo pela Universidade Presidente Antônio Carlos, Pós-graduação em Comunicação Empresarial pelo Instituto A vez do Mestre (AVM)/Universidade Cândido Mendes.
} 
Instagram e Linkedin. Trata-se de um estudo descritivo e exploratório. Foram identificados 75 periódicos. 0 acesso a cada um deles mostrou que apenas 10 (13,5\%) deles ingressaram em pelo menos uma rede social e possuíam: Twitter $(n=10)$, Facebook, $(n=8)$, Instagram $(n=2)$ e Linkedin $(n=1)$. Apenas a RP possuía as várias redes sociais estudadas. A RP tem se pautado na preocupação em oferecer educação médica continuada, em consonância com as diretrizes preconizadas pela SBP e voltada para o exercício da prática pediátrica em nosso país considerando os diferentes aspectos regionais e suas peculiaridades. $O$ foco é o residente e o pediatra que defende os direitos, a segurança, a qualidade de vida e a saúde das crianças e adolescentes brasileiros. Verificamos que ainda é insuficiente o número de revistas da área de conhecimento de pediatria do CAPES, que possuem redes sociais, apesar do crescente número de usuários que ingressam nas redes.

\section{PALAVRAS-CHAVE}

Revista eletrônica. Periódico Científico. Pediatria. Residência Pediátrica.

\section{ABSTRACT}

The Pediatric Residency journal is a four-monthly electronic publication of the Brazilian Society of Pediatrics, online since 2011, aimed at pediatric residents and related physicians, aiming to contribute to the academic education of residents and encouraging them to active participation in scientific production. This article aimed to present a history about Pediatric Residency, as well as to investigate national and international journals related to pediatrics and inserted in the CAPES platform regarding the use of social networks such as Facebook, Instagram and Linkedin. It is a descriptive and exploratory study. We identified 75 journals. Access to each showed that only $10(13.5 \%)$ of them joined at least one social network and had: Twitter $(n=10)$, Facebook, $(n=8)$, Instagram $(n=2)$ and Linkedin $(n=1)$. Only PR had the various social networks studied. The PR has been based on the concern to provide continuing medical education, in line with the guidelines recommended by the SBP and focused on the practice of pediatric practice in our country considering the different regional aspects and their peculiarities. The focus is the resident and pediatrician who defends the rights, safety, quality of life and health of Brazilian children and adolescents. We found that the number of journals in the CAPES pediatric knowledge area that have social networks is still insufficient, despite the growing number of users who join the networks.

\section{KEYWORDS}

Electronic journal. Scientific journal. Pediatrics. Pediatric Residence.

\section{INTRODUÇÃO}

\subsection{Histórico}

A revista Residência Pediátrica (RP) foi criada em 2011 como desdobramento do I Congresso de Médicos Residentes em Pediatria da Sociedade Brasileira de Pediatria (SBP), realizado no Rio de janeiro de 10 a 12 de outubro de 2010, com o objetivo de se criar uma revista eletrônica para incentivar os jovens profissionais a ler e publicar artigos científicos e de ética médica, adquirir noções de metodologia científica e exercitar a interatividade entre seus pares. 
O modelo adotado para a RP foi o de formato eletrônico, com periodicidade quadrimestral, revisado por pares (duplo cego) cujo número inicial pode ser acessado em abril de 2011 no endereço eletrônico: www.residenciapediatrica.com.br. O regulamento da RP foi aprovado em 12 de dezembro de 2012, entrando imediatamente em vigor. Inúmeras mudanças ocorreram ao longo dos anos e uma das mais significativas foi a publicação em fluxo contínuo a partir de 2018. Atualmente, além de ter qualificação CAPES, a RP é indexada em: China Knowledge Resource Integrated Database (CNKI); Diretório de Políticas Editoriais das Revistas Científicas Brasileiras (Diadorim); Google Scholar; Index Copernicus International Journals Masters List; Index Copernicus International World of Journals; Red Iberoamericana de Innovación y Conocimiento Científico (REDIB); Revistas de Livre Acesso (LivRe); Sistema Regional de Información en Línea para Revistas Científicas de América Latina, el Caribe, España y Portugal (Latindex) e Sumários de Revistas Brasileiras (Sumários.org).

As seções fixas da RP estão distribuídas em: Editorial, Artigo Original, Artigo de Revisão, Relato de Caso, Caso Clínico Interativo, Tópicos Obrigatórios em Pediatria (TOP), Ética Médica e Fique Alerta. Dependendo da disponibilidade, os números são acrescidos de outras seções a saber: Metodologia Científica, Resenha, Cartas ao Editor, Ponto de Vista e Qual é o Diagnóstico? (voltado para casos de radiologia).

A partir de 2019, os suplementos foram substituídos por artigos temáticos que passaram a ser incorporados a cada exemplar publicado.

Visando facilitar o acesso aos conteúdos e divulgar a revista para os associados da SBP, a cada número editado é enviada uma newsletter do periódico contendo informações dos novos artigos.

A estatística às diferentes seções da RP até agosto de 2019 mostrou que "Relato de Caso" e "Artigo Original totalizaram $20 \%$ e $16 \%$, respectivamente, representando as seções de maior interesse para leitura.

O corpo editorial da RP é formado por membros de renome nacional e internacional que contribuem com suas vastas experiências nas seguintes seções: Editoria Científica, Editoria Adjunta, Editoria Técnica, Conselho Editorial Executivo, Consultoria Editorial, Editoria Associada, além de uma Assistência Administrativa. Ao todo, a equipe é formada por 29 membros.

\subsection{Mídias sociais}

A visibilidade de um periódico pode ser avaliada pela sua participação em redes sociais, como o Facebook por exemplo. A presença na Word Wide Web (WWW) torna-se importante pela contribuição como fonte de informação e de atualização potencializando e disseminando o conhecimento científico (BOMFA; CASTRO, 2004). Nesse sentido, outro ponto de destaque é o número de um identificador único (DOI), registrado na base de dados da agência CrossRef. Por essa razão, os editores da RP preocupados com o acompanhamento da presença na web providenciaram o DOI para a RP. Cientes de que as estratégias de marketing devem ser aplicadas com o objetivo de estreitar cada vez mais o relacionamento com os leitores/ usuários na comunicação científica, cronologicamente, a revista RP ingressou no Facebook e no Twitter em junho de 2011, no Souncloud em março de 2016 (onde publica seus Podcasts), no Linkedin em junho de 2018, e no Instagram em março de 2019. Publicou vídeos-aulas no Youtube no período de 2011 


\section{a 2017.}

Assim, com os avanços tecnológicos e com o advento e evolução da internet, a visibilidade de um periódico tem sido cada vez mais um assunto de interesse principalmente visando a análise e o acompanhamento da sua presença online e do possível impacto acarretado.

\section{OBJETIVO}

Analisar o perfil dos periódicos nacionais e internacionais de pediatria quanto ao uso de redes sociais.

\section{PROCEDIMENTOS METODOLÓGICOS}

Trata-se de um estudo descritivo, exploratório. Foi definido como amostra da pesquisa, todos os periódicos científicos nacionais e internacionais relacionados à pediatria que estivessem inseridos na plataforma do Portal de Periódico da CAPES*. Para seleção da amostra, utilizou-se a ferramenta \&quot;buscar periódico\&quot; na área do conhecimento Ciências da Saúde e na subárea Medicina II. Pediatria. As variáveis estudadas foram: título do periódico; categoria (nacional ou internacional); tipo de publicação (eletrônica, impressa ou ambas); inserção no Facebook; Twitter e/ou Linkedin. Para obtenção das informações procedeu-se uma busca em cada um dos periódicos a fim de identificar quais as redes sociais utilizadas. Os dados foram armazenados utilizando-se o aplicativo Microsoft Excel 97-2003, sendo os resultados apresentados por números absolutos e frequências.

\section{RESULTADOS}

Foram identificados 75 periódicos. 0 acesso a cada um deles mostrou que apenas 10 (13,5\%) deles ingressaram em pelo menos uma rede social e possuíam: Twitter $(n=10)$, Facebook, $(n=8)$, Instagram ( $n=2$ ) e/ou Linkedin ( $n=1)$. Apenas a RP possuía as várias redes sociais estudadas.

O Quadro 1 apresenta os periódicos que possuem redes sociais, e que estão inseridos no CAPES, na área de pediatria. 
Quadro 1. Perfil dos periódicos de pediatria, nacionais e internacionais, inseridos no CAPES, que possuem redes sociais.

\begin{tabular}{|c|c|c|c|c|c|}
\hline NOME DO PERIÓDICO & ISSN & FACEBOOK & TWITTER & LINKEDIN & INSTAGRAM \\
\hline Acta pediátrica de México & [0186-2391] & SIM & SIM & - & $=$ \\
\hline$\underline{\text { Child care in practice }}$ & {$[1357-5279]$} & SIM & SIM & $=$ & - \\
\hline Frontiers in Pediatrics & [2296-2360] & - & SIM & - & - \\
\hline $\begin{array}{l}\text { Journal of Comprehensive } \\
\text { Pediatrics }\end{array}$ & [2251-8150] & SIM & - & - & - \\
\hline $\begin{array}{l}\text { Oxford Medical Case } \\
\text { Reports }\end{array}$ & [2053-8855] & SIM & SIM & - & - \\
\hline Paediatrica Indonesiana & [0030-9311] & - & SIM & - & - \\
\hline Pediatría atención primaria & [1139-7632] & SIM & SIM & - & SIM \\
\hline Pediatric neurology briefs & [1043-3155] & SIM & SIM & - & - \\
\hline Pediatric Quality \& Safety & [2472-0054] & - & SIM & - & - \\
\hline Residência Pediátrica & {$[2236-6814]$} & SIM & SIM & SIM & SIM \\
\hline $\begin{array}{l}\text { Twin research and human } \\
\text { genetics }\end{array}$ & [1832-4274] & - & SIM & - & - \\
\hline $\begin{array}{l}\text { Вопросы современной } \\
\text { педиатрии }\end{array}$ & [1682-5527] & SIM & - & - & - \\
\hline
\end{tabular}

Fonte: Elaboração dos autores (2019).

\section{DISCUSSÃO}

As redes sociais vêm modificando os meios de comunicação como um todo (CHARLESWORTH, 2010). O meio digital, ao contrário do meio de comunicação impresso, é interativo, e tem como característica ampliar as opções de leitura. Todos os 10 periódicos selecionados utilizavam o Twitter. Entendemos que a facilidade no envio de mensagens curtas e a possibilidade de uma leitura concisa de tópicos de interesse seja razão para o ingresso nessa rede. Apesar de o Facebook ser a rede social de maior utilização em uma escala global (CLEMENT, 2019), apenas oito periódicos a utilizavam com a finalidade de intensificar o fluxo de informações e a interatividade entre os seus usuários. Redes sociais como o Instagram e o Linkedin, tiveram pouca

\footnotetext{
${ }^{1}$ CAPES. Portal de Periódicos CAPES/MEC[website]. 2019. Disponível em: <http://www.periodicos.capes.gov.br/>. Acesso em: 30 mai 2019.
} 
adesão, ainda que por meio delas seja possível construir e publicar conteúdos relevantes e à semelhança do Facebook, podendo também ter seguidores, amplificando a divulgação de conteúdos de relevância científica. Embora não propriamente uma rede social, o Soundcloud é, na realidade, uma ferramenta que pode proporcionar praticidade no compartilhamento e divulgação de informações através de podcasts. Canais de vídeo no YouTube que abordam ciência e tecnologia ganham interesse na divulgação científica feita na internet. No entanto, ambas ferramentas ainda não têm a utilização esperada, talvez por exigirem uma estrutura de retaguarda que garanta publicações periódicas e constantes.

Um artigo publicado em 2017 mostrou que cerca de dois terços dos médicos utilizam algum tipo de mídia social por razões profissionais (HIRSCH, et al, 2017). Apesar do aumento crescente de usuários nas redes sociais e da oportunidade de auxiliar na disseminação da informação científica, ainda é pequeno o número de revistas que possuem redes sociais na área de conhecimento de pediatria. Entendemos que as novas tecnologias, principalmente no que se refere às redes sociais, canais de vídeos e podcasts podem servir como importantes ferramentas de suporte para a educação e atualização médica.

Como principal limitação do estudo exploratório, destaca-se que a amostra se restringiu às publicações de periódicos constantes em apenas uma base de dados.

\section{CONCLUSÃO}

A RP tem se pautado na preocupação em oferecer educação médica continuada, em consonância com as diretrizes preconizadas pela SBP e voltada para o exercício da prática pediátrica em nosso país considerando os diferentes aspectos regionais e suas peculiaridades. O foco é o residente e o pediatra que defende os direitos, a segurança, a qualidade de vida e a saúde das crianças e adolescentes brasileiros. Ainda é insuficiente o número de revistas da área de conhecimento de pediatria do CAPES, que possuem redes sociais, apesar do crescente número de usuários que ingressam nas redes.

\section{REFERÊNCIAS}

BOMFA, C. R. Z.; CASTRO, J. E. E. Desenvolvimento de revistas científicas em mídia digital: o caso da Revista Produção Online. Ci. Inf., Brasília, v. 33, n. 2, p. 39-48, ago. 2004. Disponível em: <http://www.scielo.br/scielo.php?script=sci_arttext\&pid=s0100-19652004000200004\&lng=en\&nrm=iso>. Acesso: em 30 mai. 2019.

CHARLESWORTH, A. Revolução Digital. São Paulo: Editora Publifolha, 2010. 72 p.

CLEMENT, J. Most popular social networks worldwide as of April 2019, ranked by number of active users (in millions). Statista, 2019. Disponível em: <https://www.statista.com/statistics/272014/global-social-networks-ranked-by-number-of-users/>. Acesso em: 10 maio 2019

HIRSCH, J. A. et al. JNIS podcasts: the early part of our journey. Journal of Neuro Interventional Surgery, v. 19, n. 2, p. 211-214, 2017. BMJ. DOI: http://dx.doi.org/ 10.1136/neurintsurg-2015$-012170$. 
SOUZA, U. de J.; CAMILO, G.; CARMO, R. C. do; SILVA, E. V. da. o uso das redes sociais pelos periódicos brasileiros de biblioteconomia e ciência da informação. Revista ACB: Biblioteconomia em Santa Catarina, Florianópolis, v. 20, n. 3, p. 584-591, set./dez., 2015. Disponível em: <https://revista.acbsc.org.br/racb/article/view/1101>. Acesso em: 14 jun 2019. 